Mathematical Research Letters 9, 449-463 (2002)

\title{
ON THE QUANTUM KAZHDAN-LUSZTIG FUNCTOR
}

\author{
Pavel I. Etingof And Adriano A. Moura
}

\section{Introduction}

One of the most exciting developments in representation theory in the recent years was the discovery of the Kazhdan-Lusztig functor [KL93a, KL93b, KL94a, KL94b], which is a tensor functor from the fusion category of representations of an affine Lie algebra to the category of representations of the corresponding quantum group, and is often an equivalence of categories. Informally speaking, this functor may be obtained by considering the monodromy of the KnizhnikZamolodchikov equations, or, equivalently, by considering exchange relations between intertwining (vertex) operators for the affine Lie algebra.

Therefore the introduction of quantized vertex operators and quantized Knizhnik-Zamolodchikov equations by Frenkel and Reshetikhin [FR92] gave rise to a hope that this remarkable functor may be q-deformed. In fact, it was implicitly suggested already in [FR92] that, since monodromy matrices of the quantized KZ equations are elliptic, the q-deformed KL functor should map the category of representations of the quantum affine algebra to the category of representations of an appropriate elliptic quantum group. However, at that time it was not clear what exactly this elliptic quantum group should be. This became much more clear after the works [Fe94a, Fe94b, FeV96, TV97]. Namely, in [Fe94a, Fe94b] elliptic quantum groups were defined using a dynamical R-matrix, in [FeV96] their representations were studied in the $s l(2)$ case, and in [TV97] it was shown that the monodromy of the quantum $\mathrm{KZ}$ equations for $\operatorname{sl}(2)$ is given by the R-matrices for these representations (this is a q-analog of the Drinfeld-Kohno theorem). More specifically, since these works and especially the later papers [JKOS99], [ABRR98],[EV99] it became transparent that the elliptic quantum group may be obtained by twisting the usual quantum affine algebra by an appropriate dynamical (or quasi-Hopf) twist ${ }^{1}$. In other words, there exists a tensor functor from the category of finite dimensional representations of the quantum affine algebra to the category of finite dimensional representations of the elliptic quantum group, which could be thought of as a q-analog of the Kazhdan-Lusztig functor (See Section 5). This idea gave rise to many interesting works, where the appropriate twist and its limiting cases were carefully studied and computed

Received March 1, 2002.

${ }^{1}$ We note that the idea of the dynamical twisting first appeared in the important paper [BBB95]. 
(see e.g. [Fra02] and references therein). Nevertheless, to the best of our knowledge, the corresponding quantum Kazhdan-Lusztig functor was never explicitly studied, and in particular it was never shown that it is fully faithful (i.e. defines an equivalence of the source category with some full subcategory of the target category).

The goal of this paper is to give a rigorous definition and to study this (essentially, previously known) functor, following the ideas of [JKOS99],[EV99]. Namely, our main result can be stated as follows.

Theorem. There exists an exact and fully faithful tensor functor $\mathcal{F}$ from the category of finite dimensional representations of the quantum affine algebra $U_{q}\left(\widehat{\mathfrak{s}} l_{n}\right)$ (with extended scalars) to the category of finite dimensional representations of Felder's elliptic quantum group $E_{\tau, \gamma / 2}\left(\widehat{\mathfrak{g}} l_{n}\right)$, for $q=e^{\pi i \gamma}$.

This theorem implies that the category of representations of the quantum affine algebra can be viewed as a full subcategory of the category of representations of the elliptic quantum group. Furthermore, it can be shown that evaluation representations of the quantum affine algebra are mapped (up to tensoring with a 1-dimensional representation) to the corresponding evaluation representations of the elliptic quantum group, which are constructed in [TV01] by pulling back finite dimensional representations of the small elliptic quantum group (with standard dynamical highest weight). Thus, the image of $\mathcal{F}$ contains (up to tensoring with 1-dimensional representations) all subquotients of tensor products of evaluation representations, and they have the same characters as the corresponding subquotients of tensor products of evaluation representations of the quantum affine algebra. Moreover, it is easy to extend the functor $\mathcal{F}$ to the category of possibly infinite dimensional representations of $U_{q}\left(\widehat{\mathfrak{s}} l_{n}\right)$, which belong to category $\mathcal{O}$ as representations of $U_{q}\left(\mathfrak{s} l_{n}\right)$. The existence of such extension implies that all such representations of $U_{q}\left(\widehat{\mathfrak{s}} l_{n}\right)$ admit an elliptic deformation. This confirms a conjecture from [TV01], where these elliptic deformations were constructed for Verma and finite dimensional modules, and conjectured to exist for any highest weight module.

\section{Representations of Quantum Dynamical R-Matrices with Spectral Parameters}

Let $\mathfrak{a}$ be a finite dimensional abelian Lie algebra over $\mathbb{C}, V$ be a finite dimensional semisimple $\mathfrak{a}$-module, and $\gamma \in \mathbb{C}^{*}$. The quantum dynamical Yang-Baxter equation with spectral parameter and step $\gamma$ is

$$
\begin{array}{r}
R^{12}\left(u_{1}-u_{2}, \lambda-\gamma h^{(3)}\right) R^{13}\left(u_{1}-u_{3}, \lambda\right) R^{23}\left(u_{2}-u_{3}, \lambda-\gamma h^{(1)}\right) \\
=R^{23}\left(u_{2}-u_{3}, \lambda\right) R^{13}\left(u_{1}-u_{3}, \lambda-\gamma h^{(2)}\right) R^{12}\left(u_{1}-u_{2}, \lambda\right)
\end{array}
$$

with respect to a meromorphic function $R: \mathbb{C} \times \mathfrak{a}^{*} \rightarrow \operatorname{End}_{\mathfrak{a}}(V \otimes V)$. The notation $h^{(i)}$ means that, for example, $R^{12}\left(u_{1}-u_{2}, \lambda-\gamma h^{(3)}\right)\left(v_{1} \otimes v_{2} \otimes v_{3}\right)=$ $\left(R\left(u_{1}-u_{2}, \lambda-\gamma \mu\right)\left(v_{1} \otimes v_{2}\right)\right) \otimes v_{3}$ if $v_{3}$ has weight $\mu$. A function $R$ is called a quantum dynamical R-matrix if it is a generically invertible solution of (1.1). 
Given a quantum dynamical R-matrix $R: \mathbb{C} \times \mathfrak{a}^{*} \rightarrow \operatorname{End}_{\mathfrak{a}}(V \otimes V)$, we denote by $\operatorname{Rep}_{\mathrm{f}}(R)$ the category of meromorphic finite dimensional representations of $R$. This category was first defined in [Fe94a, Fe94b]. It consists of pairs $\left(W, L_{W}\right)$ where $W$ is a semisimple finite dimensional $\mathfrak{a}$-module and $L_{W}$ is a meromorphic function

$$
L_{W}: \mathbb{C} \times \mathfrak{a}^{*} \rightarrow \operatorname{End}_{\mathfrak{a}}(V \otimes W)
$$

such that $L_{W}(u, \lambda)$ is (generically) invertible and satisfies

$$
\begin{array}{r}
R^{12}\left(u_{1}-u_{2}, \lambda-\gamma h^{(3)}\right) L_{W}^{13}\left(u_{1}-u_{3}, \lambda\right) L_{W}^{23}\left(u_{2}-u_{3}, \lambda-\gamma h^{(1)}\right) \\
=L_{W}^{23}\left(u_{2}-u_{3}, \lambda\right) L_{W}^{13}\left(u_{1}-u_{3}, \lambda-\gamma h^{(2)}\right) R^{12}\left(u_{1}-u_{2}, \lambda\right)
\end{array}
$$

in $\operatorname{End}_{\mathfrak{a}}(V \otimes V \otimes W)$. If $\left(W, L_{W}\right)$ and $\left(U, L_{U}\right)$ are two representations, a morphism between them is a meromorphic function $f: \mathfrak{a}^{*} \rightarrow \operatorname{Hom}_{\mathfrak{a}}(W, U)$ such that

$$
(1 \otimes f(\lambda)) L_{W}(u, \lambda)=L_{U}(u, \lambda)\left(1 \otimes f\left(\lambda-\gamma h^{(1)}\right)\right)
$$

Thus, the category $\operatorname{Rep}_{\mathrm{f}}(R)$ is linear over the field $M_{V}$ of meromorphic functions on $\mathfrak{a}^{*}$ periodic under the lattice spanned by the weights of $V$.

Example 1.1. Let $R: \mathbb{C} \times \mathfrak{a}^{*} \rightarrow \operatorname{End}_{\mathfrak{a}}(V \otimes V)$ be a quantum dynamical Rmatrix.

1. Let $W$ be any vector space and let $\mathfrak{a}$ act trivially on $W$. Then $(W, 1)$, where 1 denotes the constant function $1 \in \operatorname{End}_{\mathfrak{a}}(V \otimes W)$, is a representation of $R$ called the trivial representation associated to $W$.

2. $(V, R)$ is a representation of $R$ called the basic representation.

3. If $\left(W, L_{W}\right)$ is a representation of $R, f: \mathfrak{a}^{*} \rightarrow \operatorname{End}_{\mathfrak{a}}(W)$ is generically invertible and $L_{W}^{f}=\left(1 \otimes f(\lambda)^{-1}\right) L_{W}(u, \lambda)\left(1 \otimes f\left(\lambda-\gamma h^{(1)}\right)\right)$ then $\left(W, L_{W}^{f}\right)$ is also a representation. It is clear that $f$ provides an isomorphism from $\left(W, L_{W}^{f}\right)$ to $\left(W, L_{W}\right)$.

Example 1.2. Let $\mathfrak{a}$ be an abelian $n$-dimensional Lie algebra and $V$ be a semisimple $n$-dimensional $\mathfrak{a}$-module whose weights form a basis for $\mathfrak{a}^{*}$ (in other words, $\mathfrak{a}$ is the usual Cartan subalgebra of $\mathfrak{g l}_{n}$ acting naturally on $V=\mathbb{C}^{n}$ ). Quantum dynamical R-matrices for such $\mathfrak{a}$ and $V$ are said to be of $\mathfrak{g l}_{n}$ type [EV98, EV99]. They have the form

$$
R(u, \lambda)=\sum_{m, l} \alpha_{m, l}(u, \lambda) E_{m, m} \otimes E_{l, l}+\sum_{m \neq l} \beta_{m, l}(u, \lambda) E_{l, m} \otimes E_{m, l}
$$

The main example is the elliptic quantum dynamical R-matrix found by Felder [Fe94a, Fe94b]

$R_{\tau, \gamma}^{e l l}(u, \lambda)=\sum_{m} E_{m, m} \otimes E_{m, m}+\sum_{m \neq l} \alpha\left(u, \lambda_{m, l}\right) E_{m, m} \otimes E_{l, l}+\beta\left(u, \lambda_{m, l}\right) E_{l, m} \otimes E_{m, l}$

with

$$
\alpha(u, \lambda)=\frac{\vartheta_{1}(\lambda+\gamma ; \tau)}{\vartheta_{1}(\lambda ; \tau)} \frac{\vartheta_{1}(u ; \tau)}{\vartheta_{1}(u-\gamma ; \tau)}, \quad \beta(u, \lambda)=\frac{\vartheta_{1}(\gamma ; \tau)}{\vartheta_{1}(\lambda ; \tau)} \frac{\vartheta_{1}(u-\lambda ; \tau)}{\vartheta_{1}(u-\gamma ; \tau)}
$$


where $\lambda_{m, l}=\lambda_{m}-\lambda_{l}$, and $\vartheta_{1}$ is the standard first theta function. Felder also proposed to associate to $R_{\tau, \gamma}^{\text {ell }}$ an elliptic quantum group $E_{\tau, \gamma / 2}\left(\mathfrak{g} l_{n}\right)$, whose category of finite dimensional representations is $\operatorname{Rep}_{\mathrm{f}}\left(R_{\tau, \gamma}^{\text {ell }}\right)$.

Let $R: \mathbb{C} \times \mathfrak{a}^{*} \rightarrow \operatorname{End}_{\mathfrak{a}}(V \otimes V)$ be a quantum dynamical R-matrix. The category $\operatorname{Rep}_{\mathrm{f}}(R)$ can be endowed with a tensor product functor given by $\left(W, L_{W}\right) \odot\left(U, L_{U}\right)=\left(W \otimes U, L_{W \odot U}\right)$ where

$$
L_{W \odot U}(u, \lambda)=L_{W}^{12}\left(u, \lambda-\gamma h^{(3)}\right) L_{U}^{13}(u, \lambda)
$$

on objects and

$$
f \odot g(\lambda)=f\left(\lambda-\gamma h^{(2)}\right) \otimes g(\lambda)
$$

on morphisms. One easily checks that the first formula defines a representation, and the second one a morphism of representations. Thus, $\odot$ is a bifunctor. Moreover, it is easy to see that it defines a structure of a tensor category on $\operatorname{Rep}_{\mathrm{f}}(R)$, with unit object being the trivial representation $(\mathbb{C}, 1)$.

\section{Exchange Representations}

Let $\mathfrak{g}$ be a finite dimensional simple Lie algebra, and $\widehat{\mathfrak{g}}$ the corresponding affine Lie algebra. Let $q \in \mathbb{C}^{*},|q| \neq 1$, and let $U_{q}(\widehat{\mathfrak{g}})$ be the associated quantum affine algebra (we will use the definition given in [EFK98]). Let $\operatorname{Rep}_{\mathrm{f}}\left(U_{q}(\widehat{\mathfrak{g}})\right)$ be the category of finite dimensional (type 1) representations of $U_{q}(\widehat{\mathfrak{g}})$. For any $V \in \operatorname{Rep}_{\mathrm{f}}\left(U_{q}(\widehat{\mathfrak{g}})\right)$ and $z \in \mathbb{C}^{*}$ we can consider the shifted representation $V(z)$ where all the Chevalley generators act as before except for $e_{0}$ and $f_{0}$ which now act by $z e_{0}$ and $z^{-1} f_{0}$ respectively.

We will need the following theorem.

Theorem 2.1. (Kazhdan-Soibelman, [KS95], §5) Let $V, W \in \mathbf{R e p}_{f}\left(U_{q}(\widehat{\mathfrak{g}})\right)$, and $\mathbf{R}_{V W}(z)$ be the series obtained by evaluating the universal $R$-matrix in $V(z) \otimes W$. Then $\mathbf{R}_{V W}(z)$ is an analytic function near zero, that extends meromorphically to $\mathbb{C}$.

We will give a new proof of this result (a bit simpler than the one in [KS95]) in the appendix.

Now let $\mathfrak{h}$ denote a Cartan subalgebra of $\mathfrak{g}$, and $\widehat{\mathfrak{h}}=\mathfrak{h} \oplus \mathbb{C}$ the corresponding Cartan subalegbra in $\widehat{\mathfrak{g}}$. We denote the elements of $\widehat{\mathfrak{h}}^{*}$ by $\hat{\lambda}=(\lambda, k)$. Then we can define the fusion matrix

$$
J_{V, W}(u, \hat{\lambda}): V\left(e^{-2 \pi i u}\right) \otimes W \rightarrow V\left(e^{-2 \pi i u}\right) \otimes W,
$$

which is a formal power series in $e^{2 \pi i u}$ with coefficients being $\operatorname{End}(V \otimes W)$-valued rational functions of $q^{\left(\hat{\lambda}, \alpha_{i}\right)}$, where $\alpha_{i}$ are the simple roots. (see [ES98] and also [EFK98]). Namely, let $M_{\hat{\lambda}}$ be the Verma module over $U_{q}(\widehat{\mathfrak{g}})$ with highest weight $\hat{\lambda}$, and let $x_{\lambda}$ be its highest weight vector. Let $v \in V$ be a homogeneous vector, and $\Phi_{\hat{\lambda}}^{v}(u): M_{\hat{\lambda}} \rightarrow M_{\hat{\lambda}-\operatorname{weight}(v)} \hat{\otimes} V(z)$ be the intertwining operator such that 
the matrix coefficient $\left(x_{\hat{\lambda}-\text { weight }(v)}^{*}, \Phi_{\hat{\lambda}}^{v}(z) x_{\lambda}\right)$ equals $v$ (here $x_{\hat{\mu}}^{*}$ is the lowest weight vector of $\left.M_{\hat{\mu}}^{*}\right)$. Then for any homogeneous vectors $v \in V, w \in W$,

$$
J_{V, W}(u, \hat{\lambda})(v \otimes w):=\left(x_{\hat{\lambda}-\operatorname{weight}(v)-\operatorname{weight}(w)}^{*},\left(\Phi_{\hat{\lambda}-\operatorname{weight}(w)}^{v}\left(e^{-2 \pi i u}\right) \otimes 1\right) \Phi_{\hat{\lambda}}^{w}(1) x_{\lambda}\right) .
$$

Let $\mathrm{m}$ be the ratio of the squared lengths of long and short roots of $\mathfrak{g}(\mathrm{m}=1,2$ or 3 ), and $h^{\vee}$ be the dual Coxeter number of $\mathfrak{g}$.

Proposition 2.2. The coefficients of the series $J_{V, W}(u, \hat{\lambda})$ have an expansion into a power series in $p:=q^{-2 \mathrm{~m}\left(k+h^{\vee}\right)}$, and the sequence of these coefficients tends to 0 in the p-adic norm.

Proof. This follows from the ABRR equation for the fusion matrix [ES99] (which in our case is equivalent to the qKZ equation of Frenkel and Reshetikhin [FR92],[EFK98]).

Proposition 2.2 implies that one may consider the fusion operator $J_{V, W}(\hat{\lambda}):=$ $J_{V, W}(0, \hat{\lambda})$, which is a power series in $p$.

Proposition 2.3. The series $J_{V, W}(\hat{\lambda})$ is convergent when $|p|<<1$, and the sum of this series extends to a meromorphic function in the region $\{(\lambda, k):|p|<1\}$.

Proof. This follows from the qKZ equation [FR92],[EFK98] and Theorem 2.1.

Now define the exchange matrix with spectral parameter

$$
R_{V, W}(u, \hat{\lambda})=J_{V, W}(u, \hat{\lambda})^{-1} \mathbf{R}_{W, V}^{21}\left(e^{2 \pi i u}\right) J_{W, V}^{21}(-u, \hat{\lambda})
$$

It follows by the arguments sketched above that both $J_{V, W}(u, \hat{\lambda})$ and $R_{V, W}(u, \hat{\lambda})$ are meromorphic functions on $\mathbb{C} \times \mathfrak{U}$, where the open set $\mathfrak{U} \subset \widehat{\mathfrak{h}}^{*}$ is defined by the condition $\left|q^{-2 \mathrm{~m}\left(k+h^{\vee}\right)}\right|<1$. Moreover, these arguments actually show that the divisor of singularities of these functions is a union of hyperplanes of the form $u-b k=c,\langle x, \lambda>-b k=c$, where $b, c \in \mathbb{C}, x \in \mathfrak{h}$.

Example 2.1. Let $\mathfrak{g}=\mathfrak{s l}_{n}$. When $V=\mathbb{C}^{n}$, the formula for $R_{V, V}$ was obtained in $[\mathrm{M} 01]$.

\section{Theorem 2.4.}

1. [ES99] The fusion matrix satisfies the dynamical twist equation

$$
J_{V \otimes W, U}^{12,3}(\hat{\lambda}) J_{V, W}^{12}\left(\hat{\lambda}-h^{(3)}\right)=J_{V, W \otimes U}^{1,23}(\hat{\lambda}) J_{W, U}^{23}(\hat{\lambda})
$$

2. [ES98] For any three finite dimensional representations $V_{1}, V_{2}, V_{3}$ of $U_{q}(\widehat{\mathfrak{g}})$ the exchange matrix satisfies the quantum dynamical Yang-Baxter equation

$$
\begin{aligned}
& R_{V_{1}, V_{2}}^{12}\left(u_{1}-u_{2}, \hat{\lambda}-h^{(3)}\right) R_{V_{1}, V_{3}}^{13}\left(u_{1}-u_{3}, \hat{\lambda}\right) R_{V_{2}, V_{3}}^{23}\left(u_{2}-u_{3}, \hat{\lambda}-h^{(1)}\right) \\
& \quad=R_{V_{2}, V_{3}}^{23}\left(u_{2}-u_{3}, \hat{\lambda}\right) R_{V_{1}, V_{3}}^{13}\left(u_{1}-u_{3}, \hat{\lambda}-h^{(2)}\right) R_{V_{1}, V_{2}}^{12}\left(u_{1}-u_{2}, \hat{\lambda}\right) .
\end{aligned}
$$

In particular, for any $V \in \operatorname{Rep}_{\mathrm{f}}\left(U_{q}(\widehat{\mathfrak{g}})\right)$ the function $R_{V, V}(u, \hat{\lambda})$ is a quantum dynamical R-matrix with spectral parameter and step 1. 
Remark. Strictly speaking, the last statement of the theorem contains an abuse of terminology, since $R_{V, V}$ is a meromorphic function not on $\mathbb{C} \times \widehat{\mathfrak{h}}^{*}$, but on its open subset $\mathbb{C} \times \mathfrak{U}$. In this paper, we will ignore this terminological problem.

Let $V$ be a finite dimensional representation of $U_{q}(\widehat{\mathfrak{g}})$ and define $R_{V}=$ $R_{V, V}$. It follows from theorem 2.4 that for any $W \in \operatorname{Rep}_{\mathrm{f}}\left(U_{q}(\widehat{\mathfrak{g}})\right)$, the pair $\left(W, R_{V, W}(u, \hat{\lambda})\right)$ is a representation of $R_{V}$. It is called the exchange representation of $R_{V}$ associated to $W$.

Now let us explain the main construction of this paper. Let $V \in \operatorname{Rep}_{\mathrm{f}}\left(U_{q}(\widehat{\mathfrak{g}})\right)$. Denote by $M$ the field of meromorphic functions on $\mathfrak{U}$ and let $M_{V} \subset M$ be the subfield of functions periodic under the weights of $V$. Set $\mathcal{C}(V)=\operatorname{Rep}_{\mathrm{f}}\left(U_{q}(\widehat{\mathfrak{g}})\right) \otimes_{\mathbb{C}}$ $M_{V}$. It is easy to see then that the assignment $W \rightarrow\left(W, R_{V, W}\right)$ provides an exact, faithful functor $\mathcal{F}_{V}: \mathcal{C}(V) \rightarrow \boldsymbol{R e p}_{\mathrm{f}}\left(R_{V}\right)$.

One of our main results is the following theorem, which shows that for a nontrivial $V$, the category $\mathcal{C}(V)$ (which is obtained from $\operatorname{Rep}_{\mathrm{f}}\left(U_{q}(\widehat{\mathfrak{g}})\right.$ ) by extension of scalars) is a full subcategory in $\boldsymbol{R e p}_{\mathrm{f}}\left(R_{V}\right)$.

Theorem 2.5. The functor $\mathcal{F}_{V}: \mathcal{C}(V) \rightarrow \boldsymbol{R e p}_{f}\left(R_{V}\right)$ is a tensor functor. Furthermore, if $V$ is nontrivial, then this functor is fully faithful.

Proof. We start with the following lemma.

\section{Lemma 2.6.}

$$
R_{V, W}^{12}\left(u, \hat{\lambda}-h^{(3)}\right) R_{V, U}^{13}(u, \hat{\lambda})=J_{W, U}^{23}(\hat{\lambda})^{-1} R_{V, W \otimes U}^{1,23}(u, \hat{\lambda}) J_{W, U}^{23}\left(\hat{\lambda}-h^{(1)}\right)
$$

Proof. To shorten the notation we will write $V[u]$ instead of $V\left(e^{-2 \pi i u}\right)$. Let $\mathbf{R}_{W, V}^{21}[u]=\left.\mathcal{R}^{21}\right|_{W \otimes V[u]}$ (analogously for other pairs of indices). Then

$$
\begin{aligned}
\text { l.h.s. } & =J_{V, W}^{12}\left(u, \hat{\lambda}-h^{(3)}\right)^{-1} \mathbf{R}_{W, V}^{21}[u] J_{W, V}^{21}\left(-u, \hat{\lambda}-h^{(3)}\right) J_{V, U}^{13}(u, \hat{\lambda})^{-1} \mathbf{R}_{U, V}^{31}[u] J_{U, V}^{31}(-u, \hat{\lambda}) \\
& =J_{V[u], W}^{12}\left(\hat{\lambda}-h^{(3)}\right)^{-1} \mathbf{R}_{W, V}^{21}[u]\left(J_{W, V[u]}^{21}\left(\hat{\lambda}-h^{(3)}\right) J_{V[u], U}^{13}(\hat{\lambda})^{-1}\right) \mathbf{R}_{U, V}^{31}[u] J_{U, V[u]}^{31}(\hat{\lambda}) \\
& \stackrel{(2.1)}{=} J_{V[u], W}^{12}\left(\hat{\lambda}-h^{(3)}\right)^{-1} \mathbf{R}_{W, V}^{21}[u]\left(J_{W \otimes V[u], U}^{21,3}(\hat{\lambda})^{-1} J_{W, V[u] \otimes U}^{2,13}(\hat{\lambda})\right) \mathbf{R}_{U, V}^{31}[u] J_{U, V[u]}^{31}(\hat{\lambda}) \\
& =\left(J_{V[u], W}^{12}\left(\hat{\lambda}-h^{(3)}\right)^{-1} J_{V[u] \otimes W, U}^{12,3}(\hat{\lambda})^{-1}\right) \mathbf{R}_{W, V}^{21}[u] \mathbf{R}_{U, V}^{31}[u]\left(J_{W, U \otimes V[u]}^{21,31}(\hat{\lambda}) J_{U, V[u]}^{31}(\hat{\lambda})\right) \\
& \stackrel{(2.1)}{=}\left(J_{W, U}^{23}(\hat{\lambda})^{-1} J_{V[u], W \otimes U}^{1,23}(\hat{\lambda})^{-1}\right) \mathbf{R}_{W, V}^{21}[u] \mathbf{R}_{U, V}^{31}[u]\left(J_{W \otimes U, V[u]}^{23,1}(\hat{\lambda}) J_{W, U}^{23}\left(\hat{\lambda}-h^{(1)}\right)\right) \\
& =\text { r.h.s. }
\end{aligned}
$$

The fourth equality is clear while the last follows from the hexagon axiom for the R-matrix.

Remark. A more general version of this lemma in the non-affine situation is used in [EV00].

Now let us prove that $\mathcal{F}$ is a tensor functor. To do this, define the tensor structure $\mathcal{F}_{V}(W) \odot \mathcal{F}_{V}(U) \rightarrow \mathcal{F}_{V}(W \otimes U)$ to be given by

$$
J_{W, U}(\hat{\lambda}):\left(W, R_{V, W}\right) \odot\left(U, R_{V, U}\right) \rightarrow\left(W \otimes U, R_{V, W \otimes U}\right)
$$

It follows from Lemma 2.6 that $J_{W, U}$ is a morphism (in fact an isomorphism) and from (2.1) that it satisfies the properties of a tensor structure on a functor, as required. 
The rest of the section is dedicated to the proof of the full faithfulness of $\mathcal{F}$ in the case when $V$ is nontrivial.

Following the conventions of [EFK98], set $L_{W}^{+}(z):=\mathbf{R}_{W, V}^{21}(z)$.

Lemma 2.7. One has $\operatorname{Hom}_{U_{q}(\mathfrak{g})}(W, U)=\left\{A \in \operatorname{Hom}_{U_{q}(\mathfrak{h})}(W, U) ; L_{U}^{+}(z)^{-1}(1 \otimes\right.$ A) $L_{W}^{+}(z)$ is of zero weight in both components $\}$.

Proof. It is clear that the first space is a subspace in the second one. For the converse it is enough to prove that, if $A: W \rightarrow U$ is such that $L_{U}^{+}(z)^{-1}(1 \otimes$ A) $L_{W}^{+}(z)$ is of zero weight in both components, then $A \in \operatorname{Hom}_{U_{q}(\mathfrak{g})}(W, U)$. To do this, recall that the universal R-matrix on modules with central charge 0 can be written as

$$
\mathcal{R}=q^{\sum x_{i} \otimes x_{i}}\left(1+\sum_{i \geq 0}\left(q_{i}-q_{i}^{-1}\right) e_{i} \otimes f_{i}+\ldots\right)
$$

where $x_{i}$ form an orthonormal basis for $\mathfrak{h}$ and the remaining terms are in $U_{q}\left(\widehat{\mathfrak{n}}_{+}\right) \otimes U_{q}\left(\widehat{\mathfrak{n}}_{-}\right)$and correspond to other weights. This means that for nontrivial $V$, the condition that the expression $L_{U}^{+}(z)^{-1}(1 \otimes A) L_{W}^{+}(z)$ is of zero weight in both components implies that $A$ is of zero weight, and $\left.f_{i}\right|_{V} \otimes\left[e_{i}, A\right]=0 \forall i \geq 0$ (one just needs to look at the terms corresponding to simple roots in the expression of the universal R-matrix). It follows that $\left[e_{i}, A\right]=0$. Hence $A$ is an interwiner over $U_{q}\left(\widehat{\mathfrak{b}}_{+}\right)$. But it is proved in Proposition 4.1.3 of [KS95] that the images of $U_{q}\left(\widehat{\mathfrak{b}}_{+}\right)$and $U_{q}(\widehat{\mathfrak{g}})$ in the endomorphism algebra of any finite dimensional $U_{q}(\widehat{\mathfrak{g}})$-module are the same. Hence, $A$ is an intertwiner over the whole $U_{q}(\widehat{\mathfrak{g}})$, as desired.

Let $\mathcal{M}(W, U):=\operatorname{Hom}_{U_{q}(\mathfrak{g})}(W, U) \otimes_{\mathbb{C}} M_{V}$ be the space of morphisms between $W$ and $U$ in the category $\mathcal{C}(V)$, and let $\widetilde{\mathcal{M}}(W, U):=\mathcal{M}(W, U) \otimes_{M_{V}} M$. Lemma 2.7 implies that $\widetilde{\mathcal{M}}(W, U)$ is the space of solutions over $M$ of the system of linear algebraic equations in finitely many variables (entries of $A$ ), expressing the condition that $L_{U}^{+}(z)^{-1}(1 \otimes A) L_{W}^{+}(z)$ is of zero weight in both components.

Now let $\mathcal{N}(W, U)=\operatorname{Hom}_{\mathbf{R e p}_{\mathrm{f}}\left(R_{V}\right)}(\mathcal{F}(W), \mathcal{F}(U))$. Below we will show that the space $\tilde{\mathcal{N}}(W, U):=\mathcal{N}(W, U) \otimes_{M_{V}} M$ is contained in the space of solutions over $M$ of a deformation of this system of linear algebraic equations. This will imply that $\operatorname{dim} \mathcal{N}(W, U) \leq \operatorname{dim} \mathcal{M}(W, U)$, which immediately implies that the injective map $\mathcal{M}(W, U) \rightarrow \mathcal{N}(W, U)$ defined by $\mathcal{F}$ is actually an isomorphism, i.e., $\mathcal{F}$ is fully faithful.

To do this, we need the following lemma.

Lemma 2.8. Let $L_{W}, L_{U}$ be the exchange operators $R_{V, W}, R_{V, U}$ and $\widetilde{\mathcal{M}}^{\prime}(W, U)$ $=\left\{A(\hat{\lambda}) \in \operatorname{Hom}_{U_{q}(\mathfrak{h})}(W, U) \otimes_{\mathbb{C}} M ; L_{U}(u, \hat{\lambda})^{-1}(1 \otimes A(\hat{\lambda})) L_{W}(u, \hat{\lambda})\right.$ is of zero weight in both components $\}$. Then the natural map $\xi: \mathcal{N}(W, U) \otimes_{M_{V}} M \rightarrow$ $\widetilde{\mathcal{M}}^{\prime}(W, U)$ is injective. 
Proof. We need to show that any $M_{V^{-}}$-linearly independent set $\left\{a_{1}, \ldots, a_{m}\right\} \subset$ $\mathcal{N}(W, U)$ is also linearly independent over $M$. Assume it is false and take a counterexample with the smallest length. We can assume that $a_{m}=g_{1} a_{1}+$ $\cdots+g_{m-1} a_{m-1}$ with $g_{i} \in M$ but not all of them in $M_{V}$. Then

$$
\begin{aligned}
a_{m}\left(\hat{\lambda}-h^{(1)}\right) & =L_{U}(u, \hat{\lambda})^{-1}\left(1 \otimes a_{m}(\hat{\lambda})\right) L_{W}(u, \hat{\lambda}) \\
& =\sum g_{i}(\hat{\lambda}) L_{U}(u, \hat{\lambda})^{-1}\left(1 \otimes a_{i}(\hat{\lambda})\right) L_{W}(u, \hat{\lambda})=\sum g_{i}(\hat{\lambda}) a_{i}\left(\hat{\lambda}-h^{(1)}\right)
\end{aligned}
$$

Let $v \in V$ be homogeneous of weight $\mu$. Then, applying the last equality to $v \otimes \mathrm{Id}$, we have

$$
a_{m}(\hat{\lambda}-\mu)=\sum g_{i}(\hat{\lambda}) a_{i}(\hat{\lambda}-\mu) \Leftrightarrow a_{m}(\hat{\lambda})=\sum g_{i}(\hat{\lambda}+\mu) a_{i}(\hat{\lambda})
$$

Hence, for some $\mu, \sum\left(g_{i}(\hat{\lambda})-g_{i}(\hat{\lambda}+\mu)\right) a_{i}(\hat{\lambda})$ is a non-trivial (since not all $g_{i}$ are in $\left.M_{V}\right)$ vanishing linear combination of an even smaller length. Contradiction.

Now it remains to prove that $L_{W}(u, \hat{\lambda})$ is a deformation of $L_{W}^{+}(z)$, where $z=e^{2 \pi i u}$.

Let $\kappa=k+h^{\vee}$ and $p=q^{-2 \mathrm{~m} \kappa}$. We will write $k \rightarrow \infty$ to mean $p \rightarrow 0$ keeping $q$ fixed.

Lemma 2.9. Let $J_{V, W}(\lambda)$ be the fusion matrix corresponding to the the "finite dimensional" quantum group $U_{q}(\mathfrak{g})$. Then $\lim _{k \rightarrow \infty} J_{V, W}(u, \hat{\lambda})=J_{V, W}(\lambda)$.

Proof. Let $v$ and $w$ be homogeneous vectors in $V$ and $W$ respectively and consider the correlation function [EFK98] $\psi(z, \hat{\lambda})=z^{\Delta} J_{V, W}(u, \hat{\lambda})(v \otimes w)$ where $\Delta$ depends on the weights of $v$ and $w$ and on $\hat{\lambda}$. It is easy to see that

$$
\psi(z, \hat{\lambda})=z^{\Delta} \sum_{m \geq 0} \psi_{m}(\hat{\lambda}) z^{m}
$$

and that $\psi_{0}(\hat{\lambda})=J_{V, W}(\lambda)(v \otimes w)$. Furthermore, $\psi$ satisfies the q-KnizhnikZamolodchikov equations, from which we get

$$
\psi(p z, \hat{\lambda})=\mathbf{R}_{W, V}^{21}(p z) q_{(2)}^{\Lambda} \psi(z, \hat{\lambda})
$$

where $\Lambda$ depends only on $\lambda$ and the weights of $v, w$ (not on $k$ ). Writing $\mathbf{R}_{W, V}^{21}(z)=$ $\sum_{j \geq 0} \mathbf{R}_{j}^{21} z^{j}$ we find

$$
p^{\Delta} \sum_{m \geq 0} \psi_{m}(\hat{\lambda}) p^{m} z^{m}=\left(\sum_{j \geq 0} \mathbf{R}_{j}^{21} p^{j} z^{j}\right) q_{(2)}^{\Lambda}\left(\sum_{i \geq 0} \psi_{i}(\hat{\lambda}) z^{i}\right)
$$

Actually $p^{\Delta}$ is some constant $c$ depending only on $q, \lambda$ and the weights of $v, w$. We collect the term in $z^{l}$ in both sides to get

$$
\left(c p^{l}-\mathbf{R}_{0}^{21} q_{(2)}^{\Lambda}\right) \psi_{l}=\sum_{m=1}^{l} \mathbf{R}_{m}^{21} p^{m} q_{(2)}^{\Lambda} \psi_{l-m}
$$

from where we conclude by induction that $\psi_{l} \rightarrow 0$ as $k \rightarrow \infty$ for all $l>1$, as stated. 
Now assume that $|q|<1$ and let $\lambda \rightarrow-\infty$ mean $\operatorname{Re}\left(\lambda, \alpha_{i}\right) \rightarrow-\infty$, where $\alpha_{i}$ are the simple roots. Then, by [EV00], $J_{V, W}(\lambda) \rightarrow 1$ as $\lambda \rightarrow-\infty$, and we conclude that $L_{W}(u, \hat{\lambda}) \rightarrow \mathbf{R}_{W, V}^{21}(z)$ and, therefore, $L_{W}(u, \hat{\lambda})$ is a deformation of $L_{W}^{+}(z)$. One can proceed analogously for $|q|>1$. Theorem 2.5 is proved.

\section{Gauge Transformations}

To prove the theorem stated in the introduction, it remains to identify the category $\operatorname{Rep}_{\mathrm{f}}\left(R_{V}\right)$ for $V$ being the vector representation of $U_{q}\left(\widehat{\mathfrak{s}} l_{n}\right)$ with the category of representations of Felder's elliptic quantum group. To construct such an identification is the goal of this section. It is achieved by using the fact that the corresponding dynamical R-matrices are gauge equivalent [M01].

The concept of gauge transformations of quantum dynamical R-matrices of $\mathfrak{g l}_{n}$ type, that we briefly recall now, was introduced in [EV98].

Let $\mathfrak{h}$ be an abelian $n$-dimensional Lie algebra. A multiplicative $m$-form on $\mathfrak{h}^{*}$ is a collection of meromorphic functions $\varphi=\left\{\varphi_{a_{1}, \ldots, a_{m}}(\lambda)\right\}$ where $\left\{a_{1}, \ldots, a_{m}\right\}$ runs through all (ordered) subsets of $\{1, \ldots, n\}$ such that

$$
\varphi_{a_{1}, \ldots, a_{i+1}, a_{i}, \ldots, a_{m}}(\lambda) \varphi_{a_{1}, \ldots, a_{m}}(\lambda)=1
$$

Let $\Omega^{m}$ be the set of all multiplicative $m$-forms. With the obvious definitions one turns $\Omega^{m}$ into an abelian group where the neutral element is the constant form $\mathbf{1}_{a_{1}, \ldots, a_{m}}(\lambda)=1$.

For a given $\gamma \in \mathbb{C}^{*}$ and $s \in\{1, \ldots, n\}$ define the operator $\delta_{s}$ on the space of meromorphic functions on $\mathfrak{h}^{*}$ given by

$$
\delta_{s} f\left(\lambda_{1}, \ldots, \lambda_{n}\right)=\frac{f\left(\lambda_{1}, \ldots, \lambda_{n}\right)}{f\left(\lambda_{1}, \ldots, \lambda_{s}-\gamma, \ldots, \lambda_{n}\right)}
$$

and then the differential homomorphism $d_{\gamma}: \Omega^{m} \rightarrow \Omega^{m+1}, \varphi \mapsto d_{\gamma} \varphi$, where

$$
\left(d_{\gamma} \varphi\right)_{a_{1}, \ldots, a_{m+1}}(\lambda)=\prod_{s=1}^{m+1}\left(\delta_{a_{s}} \varphi_{a_{1}, \ldots, a_{s-1}, a_{s+1}, \ldots, a_{m+1}}(\lambda)\right)^{(-1)^{s+1}}
$$

We have $d_{\gamma}^{2} \varphi=\mathbf{1} \forall \varphi$. A form $\varphi$ is said to be $\gamma$-closed if $d_{\gamma} \varphi=\mathbf{1}$ and $\gamma$-exact if $\varphi=d_{\gamma} \psi$ for some form $\psi$. When $\gamma=1$ we just say that $\varphi$ is closed or exact and denote $d_{1}$ simply by $d$.

We list now the gauge transformations that will be important for us. Let $R$ be an R-matrix of $\mathfrak{g l}_{n}$ type and step $\gamma$ and define the following transformations

$$
R(u, \lambda) \mapsto c(u) R(u, \lambda)
$$

where $c(u)$ is a meromorphic scalar function;

$$
R(u, \lambda) \mapsto R(a u, b \lambda+\mu)
$$


where $a, b \in \mathbb{C}^{*}$ and $\mu \in \mathfrak{h}^{*}$;

$$
\begin{aligned}
R(u, \lambda) \mapsto & \sum_{m=0}^{n} \alpha_{m, m}(u, \lambda) E_{m, m} \otimes E_{m, m}+ \\
& \sum_{m \neq l} \varphi_{m, l}(\lambda) \alpha_{m, l}(u, \lambda) E_{m, m} \otimes E_{l, l}+\beta_{m, l}(u, \lambda) E_{l, m} \otimes E_{m, l}
\end{aligned}
$$

where $\varphi=\left\{\varphi_{m, l}(\lambda)\right\}$ is a $\gamma$-closed 2 -form.

It was proved in [EV98] that if $\tilde{R}$ is obtained from $R$ by these gauge transformations, then it is a quantum dynamical R-matrix of $\mathfrak{g l}_{n}$ type and step $\gamma$ (for (3.1) and (3.3)) or $\gamma / b$ (for (3.2)). It is clear that if $\tilde{R}$ is obtained from $R$ by (3.1) and (3.2), then $\boldsymbol{R e p}_{\mathrm{f}}(\tilde{R})$ is equivalent to $\boldsymbol{R e p}_{\mathrm{f}}(R)$. The following theorem, concerning gauge transformation (3.3), was proved in [EV99] in the language of bialgebroids. We repeat the proof here with the appropriate adaptations for this context.

Theorem 3.1. Let $R$ be a quantum dynamical $R$-matrix of $\mathfrak{g l}_{n}$ type and step $\gamma$ and $\varphi$ be a $\gamma$-exact 2-form. If $\tilde{R}$ is obtained from $R$ by (3.3) with this $\varphi$, then $\operatorname{Rep}_{f}(\tilde{R}) \cong \operatorname{Rep}_{f}(R)$.

Proof. Let $\varphi=d_{\gamma} \zeta$ and set $\xi=\sum_{a} \zeta_{a} E_{a, a}$. Note first that we can write

$$
\tilde{R}(u, \lambda)=\left(\xi^{(1)}\left(\lambda-\gamma h^{(2)}\right)\right)^{-1}\left(\xi^{(2)}(\lambda)\right)^{-1} R(u, \lambda) \xi^{(1)}(\lambda) \xi^{(2)}\left(\lambda-\gamma h^{(1)}\right)
$$

Then let $(W, L)$ be a representation of $R$ and set

$$
\tilde{L}(u, \lambda)=\left(\xi^{(1)}\left(\lambda-\gamma h^{(2)}\right)\right)^{-1} L(u, \lambda) \xi^{(1)}(\lambda)
$$

Now it is easy to check that the functor $(W, L) \mapsto(W, \tilde{L})$ (identity on morphisms) is an equivalence of tensor categories.

One can regard the exchange matrix $R_{\mathbb{C}^{n}}$ of example 2.1 as defined on the whole Cartan sub-algebra of $\mathfrak{g l}_{n}$. Then, if one looks at the coordinate $k$ as a parameter, it is a quantum dynamical R-matrix of $\mathfrak{g l}_{n}$ type.

Theorem 3.2. [M01] Let $R_{\tau, \gamma}^{\text {ell }}$ be the elliptic quantum dynamical R-matrix of example 1.2. Then $R_{\mathbb{C}^{n}}$ is gauge equivalent to $R_{\tau, \gamma}^{\text {ell }}$ for $\gamma=\frac{\log q}{\pi i}$ and $\tau=-\kappa \gamma=$ $-\kappa \frac{\log q}{\pi i}$, where $\kappa=k+n$.

The three gauge transformations we have listed are used in Theorem 3.2. In particular, the gauge transformation of type (3.3) used is given by the following multiplicative 2 -form

$$
\varphi_{i, j}(\lambda)=\sigma_{j, i}(\lambda / \gamma-\rho)
$$

where, for $m<l$,

$\sigma_{l, m}(\lambda)=q \frac{\Gamma_{p}\left(1+\frac{1}{\kappa}(\lambda+\rho)_{l, m}+\frac{1}{\kappa}\right)}{\Gamma_{p}\left(1+\frac{1}{\kappa}(\lambda+\rho)_{l, m}\right)} \frac{\Gamma_{p}\left(-\frac{1}{\kappa}(\lambda+\rho)_{l, m}\right)}{\Gamma_{p}\left(-\frac{1}{\kappa}(\lambda+\rho)_{l, m}+\frac{1}{\kappa}\right)}, \quad \sigma_{m, l}(\lambda)=\frac{1}{\sigma_{l, m}(\lambda)}$,

and $\Gamma_{q}$ is the q-Gamma function. 
Lemma 3.3. The multiplicative 2 -form (3.4) is $\gamma$-exact.

Proof. It is enough to show that $\tilde{\varphi}_{i, j}(\lambda)=\varphi_{i, j}(\gamma \lambda)$ is exact. Let $\xi=\left\{\xi_{j}\right\}$ be the 1 -form given by $\xi_{j}(\lambda)=\prod_{i<j} q^{\lambda_{i}}$. Then $\delta_{s} \xi_{j}(\lambda)=1$ if $s>j$ and $\delta_{s} \xi_{j}(\lambda)=q$ if $s<j$. Therefore, for $m<l,(d \xi)_{m, l}(\lambda)=q$ and $(d \xi)_{l, m}(\lambda)=q^{-1}$. In a similar way one proves that $\tilde{\varphi}=d(\xi \eta \zeta)$ where

$$
\eta_{j}(\lambda)=\prod_{i<j} \Gamma_{p}\left(\frac{\lambda_{i, j}+1}{\kappa}\right)^{-1} \quad \text { and } \quad \zeta_{j}(\lambda)=\prod_{i<j} \Gamma_{p}\left(1+\frac{\lambda_{j, i}}{\kappa}\right)^{-1}
$$

Thus, we have constructed the equivalence of categories $\mathcal{G}: \boldsymbol{R e p}_{\mathrm{f}}\left(R_{V}\right) \rightarrow$ $\operatorname{Rep}_{\mathrm{f}}\left(R_{\tau, \gamma}^{\text {ell }}\right)$, where $V=\mathbb{C}^{n}$, and hence, proved the following theorem, which is our main result.

Theorem 3.4. The functor $\mathcal{F}=\mathcal{G F}_{V}: \operatorname{Rep}_{f}\left(U_{q}\left(\widehat{\mathfrak{s l}}_{n}\right)\right) \otimes_{\mathbb{C}} M_{V} \rightarrow \operatorname{Rep}_{f}\left(R_{\tau, \gamma}^{\text {ell }}\right)$ is an exact, fully faithful tensor functor, which defines an equivalence of $\operatorname{Rep}_{f}\left(U_{q}\left(\widehat{\mathfrak{s}} l_{n}\right)\right) \otimes_{\mathbb{C}} M_{V}$ with a full subcategory of $\operatorname{Rep}_{f}\left(R_{\tau, \gamma}^{\text {ell }}\right)$.

\section{Evaluation Representations}

In this section we would like to study the action of the functor $\mathcal{F}$ on evaluation representations of $U_{q}\left(\widehat{\mathfrak{s}} l_{n}\right)$. Let $W_{\nu}(z)$ be the evaluation representation of $U_{q}\left(\widehat{\mathfrak{s}} l_{n}\right)$ with dominant integral highest weight $\nu$, evaluated at $z \in \mathbb{C}^{*}$.

Proposition 4.1. The representation $\mathcal{F}\left(W_{\nu}(z)\right)$ is isomorphic to the evaluation representation $V_{\mu}(z)$ of the elliptic quantum group defined in [TV01] (see Corollary 3.4 and Theorem 5.9), tensored with a 1-dimensional representation.

Proof. Similarly to quantum affine algebra, for elliptic quantum group there is a natural notion of a highest weight for a finite dimensional representation $W$ (it was studied in detail in [Ca01]). Namely, the highest weight is, essentially, the set of eigenvalues of the diagonal entries of the noncommutative matrix $L_{W}(u, \hat{\lambda})$ on the highest weight vector.

It is not difficult to compute highest weights of representations $\mathcal{F}\left(W_{\nu}(z)\right)$ and show that they coincide with highest weights of $V_{\nu}(z)$ up to tensoring with a 1-dimensional representation. Namely one needs to prove this statement for the fundamental representations (exterior powers), since for other representations the statement follows by taking tensor products.

To prove the statement for exterior powers, one first proves it for the vector representation $V$, in which case it is trivial, since it reduces to analyzing entries of $R_{V}$. To pass to an arbitrary exterior power, one may use fusion procedure (i.e. the fact that $\Lambda^{i} V$ is contained in the product of $i$ copies of $V$ with appropriate shifts).

Now, it was shown in [Ca01] that if two irreducible highest weight finite dimensional representations of the elliptic quantum group have the same highest weight, then they are isomorphic. This completes the proof. 
Remark. It is clear that the functor $\mathcal{F}$ extends naturally to a tensor functor $\operatorname{Rep}_{\mathcal{O}}\left(U_{q}\left(\widehat{\mathfrak{s}} l_{n}\right)\right) \otimes_{\mathbb{C}} M_{V} \rightarrow \operatorname{Rep}_{\mathcal{O}}\left(R_{\tau, \gamma}^{e l l}\right)$ between the categories $\mathcal{O}$ of representations of the quantum affine algebra, respectively the elliptic quantum group (i.e. categories of representations whose $\mathfrak{h}$-weight multiplicities are finite, and the multiplicity function is supported on a finite union of sets of the form $\mu-Q_{+}$, where $Q_{+}$is the positive cone in the root lattice of $\left.\mathfrak{g}\right)$. Namely, the construction of $\mathcal{F}$ we gave generalizes to this case in a straighforward way. Moreover, it is not hard to see that after this extension the functor remains fully faithful (the proof of full faithfulness also generalizes without significant changes). In particular, we see that any evaluation module for $U_{q}\left(\widehat{\mathfrak{s}} l_{n}\right)$ admits an elliptic deformation. This was conjectured in [TV01], and the conjecture was checked for Verma modules and finite dimensional representations.

\section{Comparison of $\mathcal{F}$ with the classical Kazhdan-Lusztig functor}

The sense in which the functor $\mathcal{F}$ is a q-analog of the Kazhdan-Lusztig functor is not straightforward, and needs to be explained.

Indeed, the Kazhdan-Lusztig functor is a functor from the fusion category of representations of an affine Kac-Moody algebra $\widehat{\mathfrak{g}}$ at some level $k$ to the category of representations of the quantum group $U_{v}(\mathfrak{g})$, where $v=e^{\left.\frac{\pi i}{\mathrm{~m}(k+h}\right)}$. On the other hand, in this paper we do not seem to be considering any q-fusion categories.

Nevertheless, there is a sense in which $\mathcal{F}$ is a q-analogue of the KazhdanLusztig functor. A two-line justification is that while the Kazhdan-Lusztig functor arises from the analysis of the monodromy of the Knizhnik-Zamolodchikov equations, the functor $\mathcal{F}$ arises in a similar way from the analysis of the monodromy of the quantum Knizhnik-Zamolodchikov equations. To some extent this is explained in [FR92] and [KS95]. However, let us give a somewhat more vivid explanation, in the special case $\mathfrak{g}=\mathfrak{s l}_{n}$. This explanation uses the small elliptic quantum group of Tarasov and Varchenko.

Recall that the classical Kazhdan-Lusztig functor $\mathcal{F}_{0}$, essentially, does the following. Given a finite dimensional representation $V$ of $\mathfrak{g}$ and a (generic) level $k$, it introduces on $V$ an action of the quantum group $U_{v}(\mathfrak{g})$, with $v=e^{\left.\frac{\pi i}{\operatorname{m(k+h} v}\right)}$. More precisely, one first uses the exchange construction for $\widehat{\mathfrak{g}}$ at level $k$ to turn $V$ into a representation of the exchange dynamical quantum group $F_{v}(G), G=S L_{n}$ (see [EV99]), and then turns it into a representation of $U_{v}(\mathfrak{g})$ by sending the dynamical parameter to infinity.

On the other hand, given a (generic) level $k$ and a finite dimensional representation $V$ of $U_{q}(\mathfrak{g})$, the functor $\mathcal{F}$ introduces on $V$ an action of the elliptic quantum group $E_{\tau, \gamma / 2}(\mathfrak{g})$, with $\tau=-\frac{\left(k+h^{\vee}\right) \log q}{\pi i}$, and $\gamma=\frac{\log q}{\pi i}$ (by first affinizing $V$ and then applying the functor $\mathcal{F}$ ).

Now, one may check using modular invariance of theta-functions that $E_{\tau, \gamma / 2}(\mathfrak{g})$ is isomorphic to $E_{-1 / \tau, \gamma / 2 \tau}(\mathfrak{g})$ (the corresponding dynamical R-matrices are gauge equivalent). Thus, $V$ becomes a representation of $E_{-1 / \tau, \gamma / 2 \tau}(\mathfrak{g})$. This 
representation is easily seen to be an evaluation representation, i.e., it factors through the small elliptic quantum group $e_{-1 / \tau, \gamma / 2 \tau}(\mathfrak{g})$ ([TV01]).

Finally, we claim that if $k$ is fixed then the algebra $e_{-1 / \tau, \gamma / 2 \tau}(\mathfrak{g})$ is a qdeformation of the exchange dynamical quantum group $F_{v}(G)$ (which allows us to regard $\mathcal{F}$ as a q-deformation of $\mathcal{F}_{0}$ ). Indeed, it is easy to check that as $\omega \rightarrow \infty, e_{\omega, a}(\mathfrak{g})$ degenerates into $F_{e^{-2 \pi i a}}(G)$. Since we have $-1 / \tau=\frac{\pi i}{\left(k+h^{\vee}\right) \log q}$, and $\gamma / 2 \tau=-\frac{1}{2\left(k+h^{\vee}\right)}$, the claim follows.

\section{Appendix A. Meromorphicity of the R-matrix}

In this appendix we give a new proof of Theorem 2.1.

Let $U$ be a neighborhood of zero in $\mathbb{C}^{n}$, and $G: U \rightarrow \mathbb{C}^{n}$ be an analytic mapping, such that $G(0)=0$. Let $p \in \mathbb{C},|p|>1$.

Lemma A.1. Let $f \in z \mathbb{C}^{n}[[z]]$ be a formal solution to the difference equation

$$
f(p z)=G(f(z))
$$

Then $f$ converges to an analytic function in some neighborhood of zero.

Proof. Let $f(z)=\sum_{n \geq 1} f_{n} z^{n}$, and $G(y)=\sum_{n \geq 1} g_{n} y^{n}$, where $g_{n}$ are multilinear operators. Since $G$ is analytic at zero, there exists $A>0$ such that the norms of the coefficients are estimated by $\left|g_{n}\right|<A^{n}$ for all $n$.

Let $k_{0}$ be so big that for $k \geq k_{0}$ one has $\left|\left(p^{k}-g_{1}\right)^{-1}\right|<2|p|^{-k}$, and $2 A<|p|^{k / 2}$. Let $C$ be such that $\left|f_{k}\right|<C$ for $k<k_{0}$, and let $B>\max \left(1, \frac{A C}{|p|^{1 / 2}-1}\right)$.

Let us prove by induction in $k$ that $\left|f_{k}\right|<C B^{k-1}$. The statement is clear for $k<k_{0}$ (as $B>1$ ). Assume that the statement is known for all $n<k$, for some $k \geq k_{0}$, and prove it for $k$. We have

$$
f_{k}=\left(p^{k}-g_{1}\right)^{-1} \sum_{1<r \leq k} \sum_{i_{1}, \ldots, i_{r}: \Sigma} g_{i_{j}=k}\left(f_{i_{1}}, \ldots, f_{i_{r}}\right)
$$

Now, $\left|g_{r}\left(f_{i_{1}}, \ldots, f_{i_{r}}\right)\right| \leq\left|g_{r}\right| \prod_{l=1}^{r}\left|f_{i_{l}}\right|<(A C)^{r} B^{k-r}$, and the number of terms with $g_{r}$ is dominated by the binomial coefficient $\left(\begin{array}{l}k-1 \\ r-1\end{array}\right)\left(\right.$ as $\left.i_{j} \geq 1, r>1\right)$. Thus, we have

$$
\left|f_{k}\right|<2(A C / B)(1+A C / B)^{k-1}|p|^{-k} B^{k}<C B^{k-1},
$$

since $2 A<|p|^{k / 2}$ and $1+A C / B<|p|^{1 / 2}$. The inductive step is proved.

This implies that $f(z)$ converges for small $z$. We are done.

Now we proceed with the proof of Theorem 2.1. Recall the crossing symmetry condition (equation (9.19) of [EFK98])

$$
\left.\left(\left(\left(\mathbf{R}_{V, W}(z)^{-1}\right)^{t_{1}}\right)^{-1}\right)^{t_{1}}\right)=\left(q^{2 \rho} \otimes 1\right) \mathbf{R}_{V, W}\left(z q^{2 \mathrm{~m} h^{\vee}}\right)\left(q^{-2 \rho} \otimes 1\right)
$$

where $t_{1}$ is transposition in the first component and $\rho$ is the half-sum of positive roots of $\mathfrak{g}$. It is valid for any finite dimensional representations. If $|q| \neq 1$, it can be regarded as an equation of the type $f(p z)=G(f(z))$ considered above. Namely, $f$ takes values in the space of endomorphisms of $V \otimes W$, 
$p=q^{ \pm 2 \mathrm{~m} h^{\vee}}, G(X)=\left(\left(\left(X^{-1}\right)^{t_{1}}\right)^{-1}\right)^{t_{1}}$, and the origin in $\operatorname{End}(V \otimes W)$ is at the point $X=\mathbf{R}_{V, W}(0)$, which is a fixed point of $G$. Thus, by Lemma A.1, the function $\mathbf{R}_{V, W}(z)$ is analytic near zero. Then the meromorphicity in the whole complex plane follows immediately from the difference equation (A.1) and the fact that in our case $G$ is a birational isomorphism.

Remark. It is clear that the same result and proof applies to $V, W$ being in category $\mathcal{O}$ as $U_{q}(g)$ modules (since the R-matrix has zero weight, and the weight spaces of $V \otimes W$ are finite dimensional).

\section{Acknowledgments}

The first author is grateful to A. Varchenko for many useful discussions about the subject matter of this paper, and to G. Felder for explaining the content of the work [Ca01]. The work of the first author was partially supported by the NSF grant DMS-9988796, and was done in part for the Clay Mathematics Institute. The second author is grateful to MIT for hospitality. The Ph.D. studies of the second author, during which this work was accomplished, are supported by FAPESP (99/11600-0), and his visit to MIT is supported by CAPES (0365/017), Brazil.

\section{References}

[ABRR98] D. Arnaudon, E. Buffenoir, E. Ragoucy, Ph. Roche, Universal solutions of quantum dynamical Yang-Baxter equations, Lett. Math. Phys. 44 (1998), 201-214.

[BBB95] O. Babelon, D. Bernard, E. Billey, A quasi-Hopf algebra interpretation of quantum 3-j and 6-j symbols and difference equations, Phys. Lett. B 375 (1996), 89-97.

[Ca01] A. Cavalli, Ph.D. thesis, ETH, Zurich, 2001.

[EFK98] P. Etingof, I. Frenkel, A. Kirillov Jr., Lectures on representation theory and Knizhnik-Zamolodchikov equations, Mathematical Surveys and Monographs, 58. American Mathematical Society, Providence, RI, 1998.

[ES98] P. Etingof, O. Schiffmann, On highest weight modules over elliptic quantum groups, preprint: QA/9809161

[ES99] Lectures on the dynamical Yang-Baxter equations, preprint, 1999: QA/9908064

[EV98] P. Etingof, A. Varchenko, Solutions of the quantum dynamical Yang-Baxter equation and dynamical quantum groups, Comm. Math. Phys, 196 (1998), 591-640.

[EV99] _ Exchange dynamical quantum groups, Comm. Math. Phys 205 (1999), $19-52$.

[EV00] - Traces of intertwiners for quantum groups and difference equations. I. Duke Math. J. 104 (2000), 391-432.

[Fe94a] G. Felder, Conformal field theory and integrable systems associated to elliptic curves, Proceedings of the International Congress of Mathematicians, Vol. 1, 2 (Zrich, 1994), 1247-1255, Birkhäuser, Basel, 1995.

[Fe94b] Elliptic quantum groups, 11th International Congress of Mathematical Physics (Paris, 1994), 211-218, International Press, Cambridge, MA, 1995.

[FeV96] G. Felder, A. Varchenko, On representations of the elliptic quantum group $e_{\tau, \gamma}\left(\mathfrak{s l}_{2}\right)$, preprint: q-alg/9601003

[Fra02] L. Frappat, Quantum elliptic algebras and double Yangians, preprint: QA/0201245

[FR92] I. Frenkel, N. Reshetikhin, Quantum affine algebras and holonomic difference equations, Comm. Math. Phys. 146 (1992), 1-60. 
[JKOS99] M. A. Jimbo, H. Konno, S. Odake, J. Shiraishi, Quasi-Hopf twistors for elliptic quantum groups, Transformation Groups 4 (1999), 303-327.

[KL93a] D. Kazhdan, G. Lusztig, Tensor structures arising from affine Lie algebras. I. J. Amer. Math. Soc. 6 (1993), 905-947.

[KL93b] , Tensor structures arising from affine Lie algebras. II. J. Amer. Math. Soc. 6 (1993), 949-1011.

[KL94a] , Tensor structures arising from affine Lie algebras. III. J. Amer. Math. Soc. 7 (1994), 335-381.

[KL94b] Tensor structures arising from affine Lie algebras. IV. J. Amer. Math. Soc. 7 (1994) 383-453.

[KS95] D. Kazhdan, Y. Soibelman, Representations of quantum affine algebras, Selecta Math. (N.S.) 1 (1995), 537-595.

[M01] A. Moura, Elliptic dynamical R-matrices from the monodromy of the q-KnizhnikZamolodchikov equations for the standard representation of $U_{q}\left(\tilde{\mathfrak{s l}}_{n+1}\right)$, preprint: rt/0112145

[TV97] V. Tarasov, A. Varchenko, Geometry of q-hypergeometric functions, quantum affine algebras and elliptic quantum groups, Astérisque No. 246, 1997.

[TV01] , Small elliptic quantum group $e_{\tau, \gamma}\left(\mathfrak{s l}_{n}\right)$, Mosc. Math. J. 1 (2001), 243-286, 303-304.

Mit, 77 Massachusetts Avenue 2-176, Cambridge, MA 02139-4307, U.S.A.

E-mail address: etingof@math.mit.edu

IMECC - UNiCAMP, Caixa Postal : 6065, CEP : 13083-970, Campinas, SP - Brasil. E-mail address: adrianoam@ime.unicamp.br 